\title{
Identify eight aspects of ergonomics to determine the improvement of human-machine interaction work (case studies in manufacturing industry)
}

\author{
Wahyu Susihono ${ }^{1 *}$,Tania Anggi Saputri ${ }^{2}$ \\ ${ }^{1,}$, Industrial Engineering Department, Engineering Faculty, University of Sultan Ageng Tirtayasa, \\ Banten, Indonesia
}

\begin{abstract}
Manufacturing Industry is one of the industrial activities in Indonesia, manufacturing industry is an industry with main activities is to change raw materials, components, or other parts into goods which is according to company specifications standards. In the production floor, activity in the manufacturing industry, the workers have different job specifications with each other. Some works consist of human-machine interaction is found by the activity between workers and lathe machine, welding maching, milling machine, frais machine, and others. The manufacturing industry will increase its ability to serve a variety of better quality products caused by the desire or encouragement of the customers. In general, according to the increase of corporate targets, its also need improvement from the aspect of work performance. To obtain a description of the proposed improvement based on human performance, it is necessary to identify the eight aspects of ergonomics include the consumption of nutritionalfor workers (energy), muscle power, body posture, environment, time conditions, social conditions, information conditions, and human machine interaction. This research use cross sectional method approach that is research done at one time, no follw up, to find the correlation between independent variable (risk factor) with dependent variable (effect). The conclusion of this research is needed nutrition intake or nutrition to recover the workers, it is necessary to design facilities such as manufacturing aids to reduce the use of excess muscle or appropriate technology (TTG). After the application of TTG (Appropriate Technology) to reduce the excessive use of muscle to the workers, the company should provide the nutritional intake accordance with workload of employees in the manufacturing industry
\end{abstract}

\section{Introduction}

Ergonomics is a component of activities which is within a broad scope, among others, include the reciprocal harmonixation of work on labor for efficiency and comfort of work

* Corresponding author: pmy_wahyu@yahoo.co.id 
$[1,2]$. The target of ergonomics is the entire workforce of both the modern sector as well as in the traditional and informal sectors. Basically all activities that use man in it will always be related to ergonomics because of its relation to the correct working procedure.

The main problem in activity on the production floor of manufacturing industry, is still very complex. The need for applying the ideal conditions of healthy manufacturing industry still needs to be supported by several factors that need to be improved. One of the factors, begin from the division of labor which is unwell organized, until the adoption of technology that still needs attention. Safety and health issues of workers is still far from ideal conditions. As example in production floor activities to produce goods, until nowadays required evaluation on worker's activities start from applying of good working methods, work nutrition, and the use of work tools. Other condition of concern are musculoskeletal complaints by workers during work on this production plant, because workers bend their body during work, and also hot on production plant can make workers do not feel comfortable while working.

From the initial observation there is still a lot of work done without considering the aspects of ergonomics either from the method of work or the facilities used. The ergonomics approach offers an approach that can evaluate 8 aspects of ergonomics within the framework of improvement of work methods. Eight aspects of ergonomics in the settlement of ergonomic poblems include: energy or nutritional status (nutrition), musculosceletal energy use, work posture, time, social conditions, information conditions, and machine interface $[3,4,5]$. Problems are identified together to gain success in improving performance of work that can be shared.

Manufacturing activities that exist in this manufactuing industry are: manufacture of spare parts to fulfill orders from other companies, receiving machine service, and fixing the construction objects. There are 40 workers in it including operators and administrative staff with working time of 8 hours of work and 1 hour breaktime for 6 work days.

In this manufacturing industry, there are still workers' muscle complaints that become one of the main factors causing the decline of work performance in this industrial sector. Based on this problem, it is necessary to conduct an evaluation study of 8 aspects of ergonomics, so as to provide alternatives in the form of: (1) the availability of appropriate technology (TTG) on production plant, and (2) the evaluation of work nutrition as work recovery

\section{Methods}

This research was conducted on manufacturing industry studies on the operator of the process of turning, welding, fraising, and milling. This research was conducted by cross sectional, on estudy and one time, no follow-up to find the correlation between independent variable (risk factor), and dependent factor (effect).

The stages of this research are preliminary study, identification of 8 aspects of ergonomics, identification of appropriate technology (TTG), operational nutrition assessment of operator, suggesting for improvement, giving thr conclution and suggection as the final.

\section{Results And Discussion}

Based on the results of preliminary study and observations that have been done on the manufacturing industry on the identification of 8 aspects of ergonomics is known as follows. 


\subsection{Nutrition}

On the production floor of manufacturing industry has a hot environment so the consumption of carbohydrates and minerals is dominant [3]. The fulfillment of nutrients is just eating and drinking that has no rules regarding the criteria of nutritional intake. The purpose of providing nutrition at both active and lunch breaks is for energu recovery after completion of work. So the energy released by the body is proportional to the nutritional intake that should be received by the body. Supplementary nutriion or nutrition to workers (average age 37.73 and weight $64 \mathrm{~kg}$ ) can be calculated to find the value of the adequacy of nutrition work. The amount of additional nutrition work is calculated according to the needs of calories by the workers as known as follows: a) the average worker;s wight, b) the value of the Basal Metabolism Rate (BMR), c) the energy adequacy rate based on the level of physical activity, d) Specific Dynamic Action or $10 \%$ intake. Intake for additional nutrition or nutrition at the time of active rest is calculated by $10 \%$ of the total calorific value. The calculation of the overall worker calorie requirement is as follows.

a. Basal Metabolisme Rate (BMR) is 11,6 weight $+876=1618.4$ calories.

b. The total energy requirement or energy adequacy based on the level of physical activity is $1.88 \times 1618,4=3042.56$ calories.

c. Specific Dynamic Action or intake (10\%) is 3346.85 calories.

In order to meet the nutritional need of workers, the overall worker calorie requirement of 3346,85 caloriea is a reference for the division of nutritional adequacy and the food menu from provided in the morning and afternoon. If the food intake is lowered, the worker will experience malnutrition and potentially adverse effect on the health condition of the employee, however, the exess of food intake such as provide high calorie also effect the incidence of excessive body fat, and if not balanced with the activity which can burn the body's calories.

\subsection{Muscle power}

Muscle expenditure on some tasks performed by operator included in activities that require great muscle power. For example in material transport activities in station. In addition, other activities such as welding, and turning do not require large muscle power except when the activity set up raw materials to the machine.

\subsection{Body posture}

Some tasks performed by operators are known not ergonomic, for example, the operators who use the lathe machine while doing set up of the machine, with a bent posture can cause injury to the backbone. Here are the pictures of worker's posture while working.

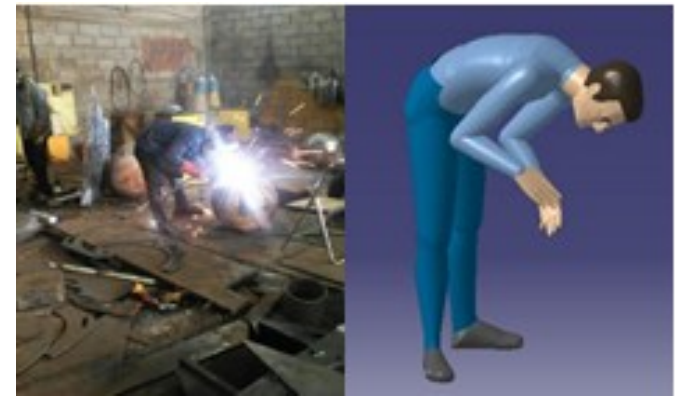

(a)

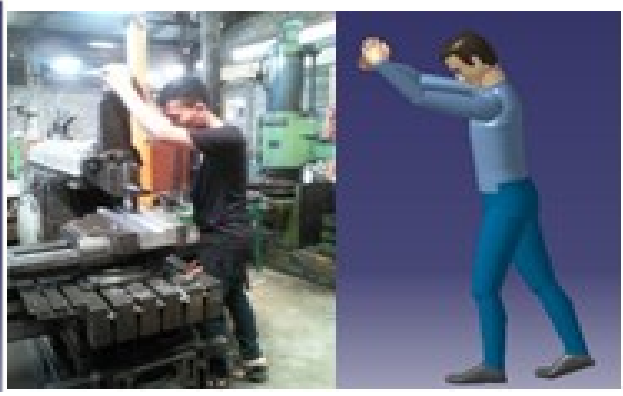

(b)

Fig. 1. (a) Body posture at welding, (b) Body posture at frais 
Based on figure 1 (a), it shows the posture when workers welding the objects is bending. This can cause back muscle injury. Based on the figure 1(b), shows that the work posture on the frais stastion, there is a bad poture such as lifting of the shoulders and elbows upward when doing the process of frais on the workpiece. This can cause arm muscle injury.

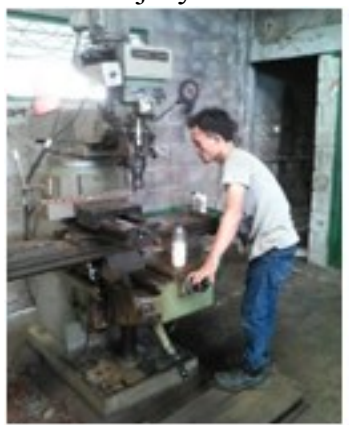

(a)

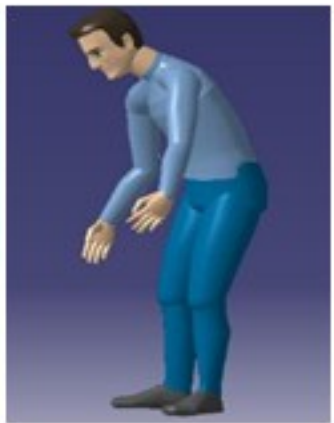

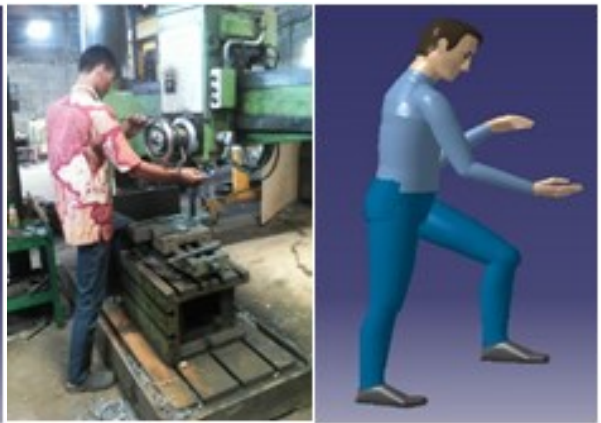

(b)

Fig. 2. (a) Body posture at hand milling station, (b) Body posture at milling station

Based on the figure 2 (a), shows the body posture of the operator while doing the milling process is slightly bent and shoulder lifted. This can cause neck and spine injuries. Based on the figure 2(b), the posture of milling operator with lifted leg. This isn't belongs to the healthy posture (the body working in a natural position or straight leg).

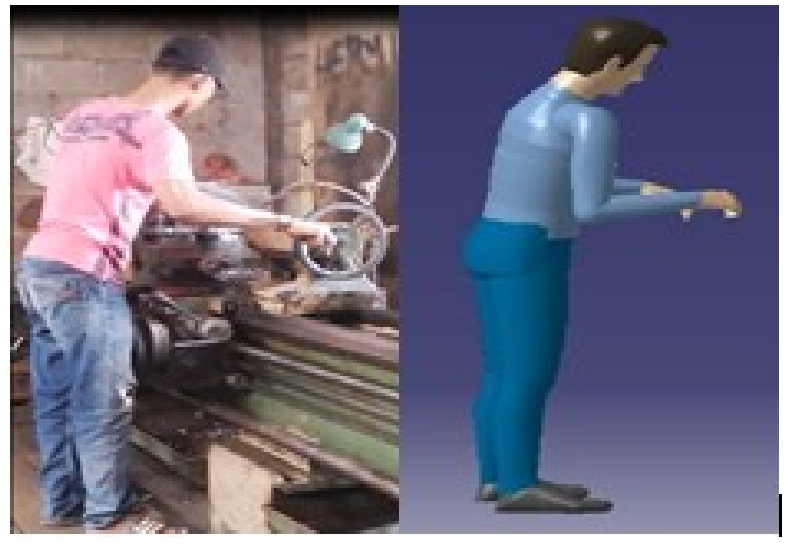

Fig. 3. Postur kerja stasiun bubut

Based on figure 3, the posture of the operator at the process of lathing is with raised shoulders. This does not correspind to the supposed working position of the body working with normal shoulder position.

\subsection{Environment}

\subsubsection{Welding Station}

Based onthe results of physical environmental data at welding stations, it is known that the average of humidity is $64 \%$, if this compared with the threshold value according to the desicion of the Minister of Health RI 1405/MENKES/SK/XI/2002 is 40-60\%. Excessively high humidity accompanied by high environmental temperatures can increase heart rate and accelerate the evaporation of sweat ehich affects worker will feel prolonged thirst $[6,7]$. The 
average value of the temperature of this manufacturing industry is $32.9^{\circ} \mathrm{C}$, and when compared with the thershold value according to the desicion of Minister of Helath RI No. 1405/MENKES/SK/XI/2002 can be said this condition have exceeded the limit of thershold value for medium type of work is $26.7{ }^{\circ} \mathrm{C}$ and the heavy work load is $25^{\circ} \mathrm{C}$. The average noise rating of the manufacturing industry is $43.42 \mathrm{~dB}$, the noise thershold value with 8 hours of exposure time in one day is $85 \mathrm{~dB}$, so it can be categorized as safe.

\subsubsection{Frais Station}

Based on the results of the temperature at the frais station, the average lighting on this station is 258.2 lux, if this results is compared with lighting thershold value, then it can be categorized as safe. The average of humidity of this manufacturing industry is $58 \%$, this result if compared with thershold value at frais station can be categorized as safe. The average of temperature value of this station is $33.5^{\circ} \mathrm{C}$, when this results is compared with the optimal working temperature of thershold value $26.7{ }^{\circ} \mathrm{C}$ for moderate work, and $25^{\circ} \mathrm{C}$ for heavy work, so this average result can be categorized has exceeded the safe limit. The average noise output at frais station is $78.76 \mathrm{~dB}$, when compared with thershold value that is $85 \mathrm{~dB}$ with exposure time is 8 hours, so it can be categorized as safe.

\subsubsection{Milling Station}

Based on the average result of the lighting on the production floor of the milling station in manufacturing industry is known with the result is 199,2lux, if compared with the lighting thershold value on Minister of Health RI No. 1405/MENKES/SK/XI/2002 is 100 lux, so the average of these illustrations can be categorized as safe. The average of humidity in manufacturing industry is $60,8 \%$, when compared with thershold value is $40-60 \%$, then this result can be said to have exceeded the limit. The average temperature at the milling station is $33.5^{\circ} \mathrm{C}$, while the thershold value for moderate work is $26.7^{\circ} \mathrm{C}$, and for heavy work is 25 ${ }^{\circ} \mathrm{C}$, the average of temperature can be categorized to have exceeded the thershold limit. The average noise rating is $81.06 \mathrm{~dB}$, this results if compared to thershold value of noise is $85 \mathrm{~dB}$, so this average value can be categorized as safe.

\subsubsection{Turning Station}

Based on the average results of lighting on the production floor of turning station in manufacturing industry is known with the result of 115,4lux, if it compared with lighting thershold value in Minister of Health RI No. 1405/MENKES/SK/XI/2002 is 100 lux, so the average of these results can be categorized as safe. The average of humidity in manufacturing industry is $58 \%$, if compared with humidity thershold value is $40-60 \%$ then this results can be categorized as safe. The average temperature at the milling station is $33.3^{\circ} \mathrm{C}$, while the thershold value for moderate work is $26.7^{\circ} \mathrm{C}$, and the thershold for heavy work is $25^{\circ} \mathrm{C}$, the average of this temperature can be categorized as exceeded the thershold limit. The average noise is $81,66 \mathrm{~dB}$, if this results is compared to noise thershold value is $85 \mathrm{~dB}$, so this average can be categorized as safe.

\subsection{Time Conditions}

Effort to restore power certainly require rest time to release tired and reduce the workload $[8,9]$. Working in hours at manufacturing industry is accordance with the rules applied by government, the optimal working is eight hours with an hour of break time during the day. 


\subsection{Social}

The social life at work can be the interaction between workers, family, and company $[9,10,11]$. In the manufacturing industry has a habbit of briefing among workers in the morning before work begins. This is done because the type of work is changing accorrding to demand so the communication between operational managers and machine operators is must. Unfortunately, in this manufacturing industry is still lacking awareness of workers to use the Safety Equipment on each job so that the accidents is probably happen.

\subsection{Information}

The information condition in the production floor such as: applying the display in the work area enable the distribution of information to avoid mistakes of work's procedure, knowing the location of the retievl and the re-laying of goods, as well minimize the questioning to the operational manager.

\subsection{Man-Machine Inteface}

Human-machine interaction in the manufacturing industry, shows by how the workers using the equipment or work system that focusing on man to adjust their work (fitting mn to the job). For examples, at the turning station, operator uses beam as footrest so that operator can do their set up carefully. Then, for lifting the heavy workpiece, the company has provided manual crane as a tool for material transportation. Based on the result of posture analysis using CATIA, the score of each operator of machining process as follows.

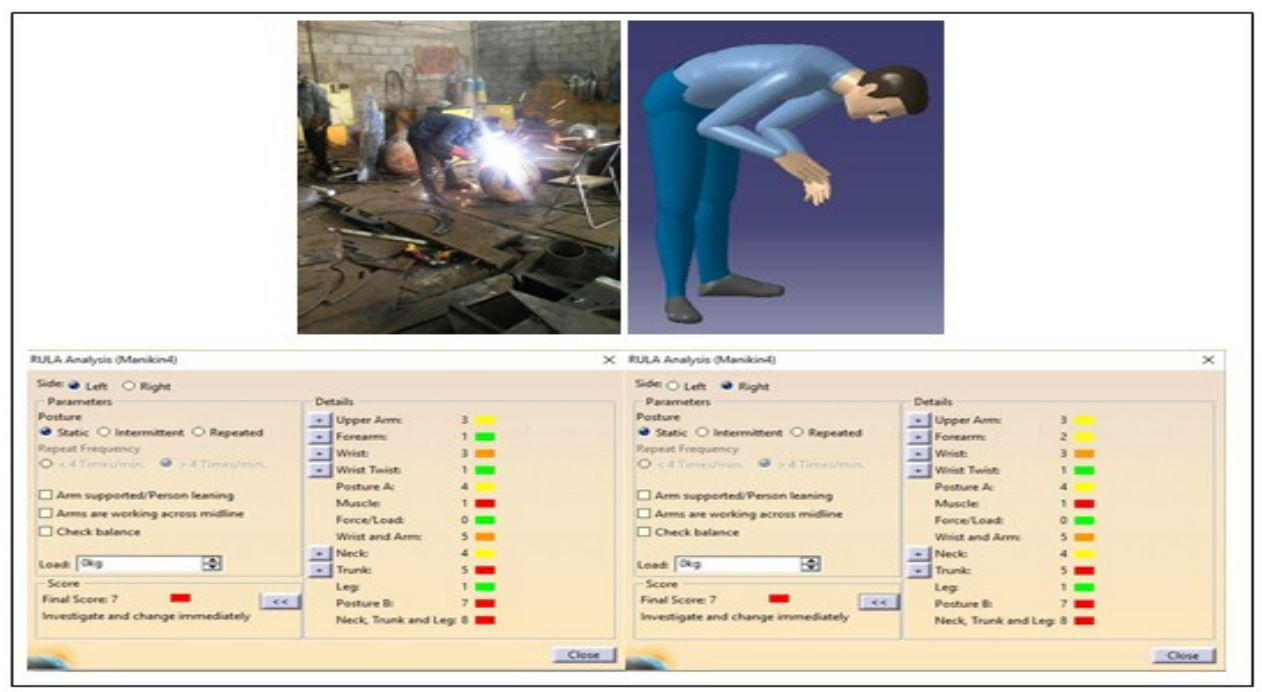

Fig. 4. RULA Score of Welding Operator

Based on the posture analyze onthe welding process, the RULA Score is seven as shown in figure 4, which is categorized as high risk level. In thiis condition, needed the repair action at work system immediately, because this situation can causes the injury of worker. In the frais process, the RULA Score of operator is 5, as shown in figure 2(a). This score can be categorized the medium level, in this condition required improvement of work system in the future. Than at the lathe process the RULA score is as shown in figure 2(b). This score shows the medium level risk in this condition so required repair of work system in the future. Based on the results of the RULA score, it is known that highest and most 
dangerous scores are in the welding operation where the posture is bent, so that's why can cause injury at work if not repair well. Therefore, the the appropriate technoogy for this manufacturing industry is located in welding station. This kind of aprropriate technology is adjustable welding table with hydraulic, so that the height of object could be adjusted by the height of operator. This following is a drawing of the hydraulic welding table for welding station.

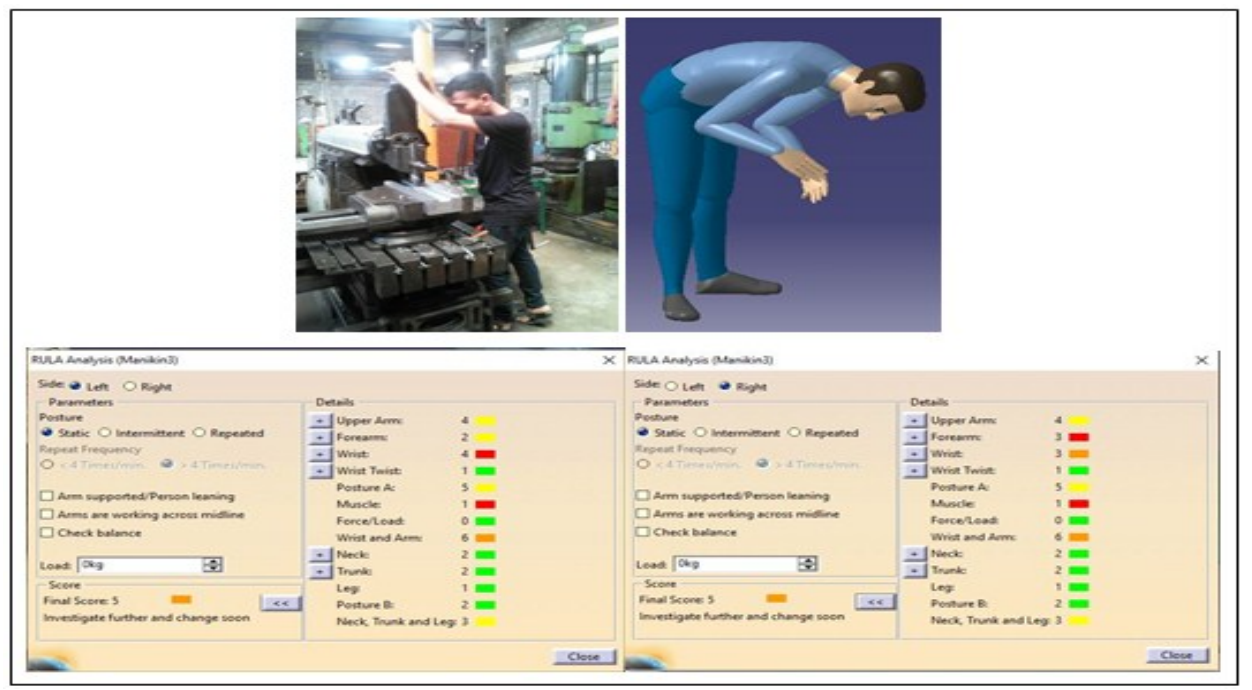

Fig. 5. RULA Score of frais operator

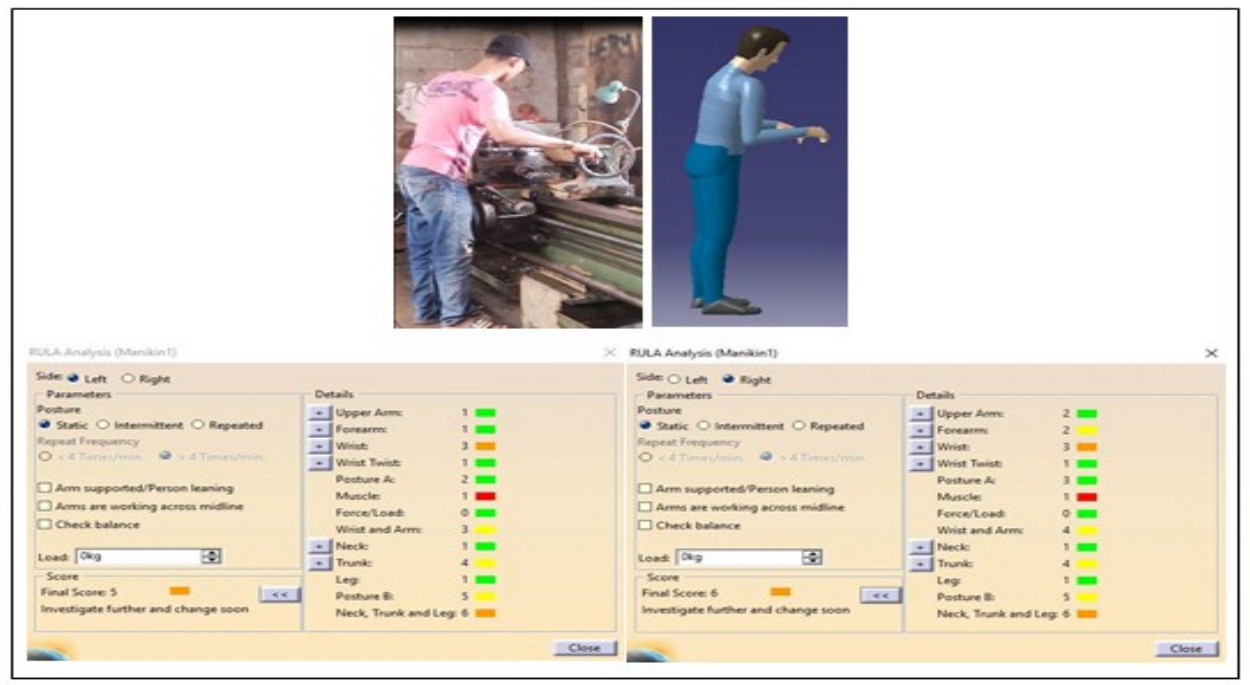

Fig. 6. RULA Score of turning operator

This technology has a hydraulic working principle with a lever rotation control that function is to adjust the height of table with the height of operator and the height of the workpiece. This hydraulic table has a thickness $8 \mathrm{~cm}$, width $1 \mathrm{~m}$, and length $2 \mathrm{~m}$. This hydraulic table is designed with refractory material 


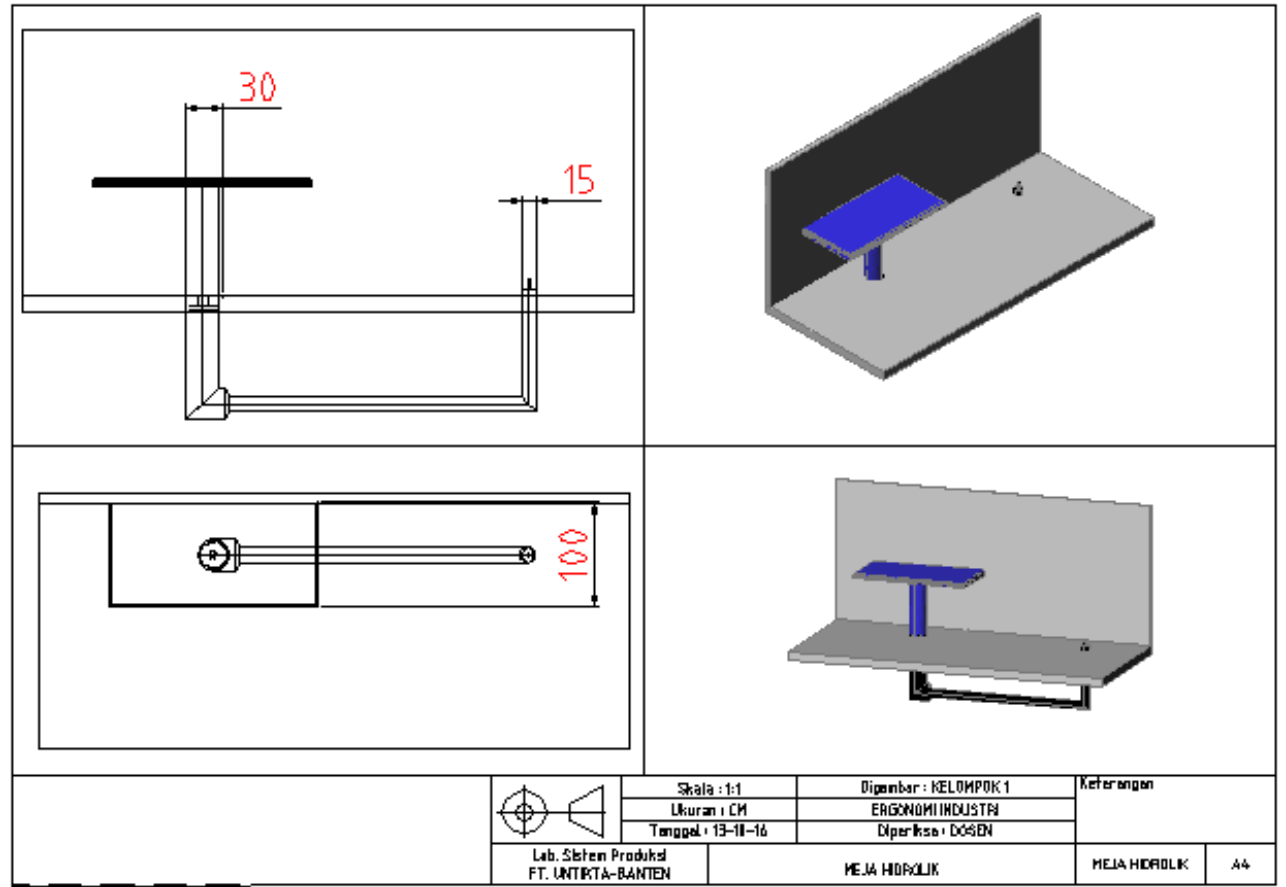

Fig. 7. Hydraulic welding table.

This hydraulic welding table provides technical benefits that facilitate the operator in performing the process on the workpiece with varying heights, then the benefits in terms of ergonomics is to improve the health posture of operator thereby reducing complaints musculosceletal disorders, then economically can increase the productivity of the company thus increasing revenue of the company, in addition to the economic terms of this tool also does not reqiure expensive investment because of its simple working principle and do not use advanced technology.

\section{Conclusion}

The following is the conclusion and suggestion of the discussion and analysis that has been done:

1. Based on 8 aspects od ergonomics, the aspects that need to be evaluated are: the oprator's posture tends was harmful to the back muscles and the worker's arms so that it is necessary to adjust the anthropometry of the worker. In the work area need a wellorganized layout and does not exceed the thershold value. The evaluation on the habbit of this manufacturing industry is by applying the personal protective equipment (PPE) while working as a safety and health management system.

2. Basal Metabolisme Rate (BMR) is 1618.4 calories, while total energy adequacy value based on physical activity is 3402,56 calories. So the obtained Spesific Dynamic Action or intake $(10 \%)$ is 3346.85 calories.

3. Appropriate technology results are used in welding stations (the most dangerous by RULA Analysis score) this technology is using a hydraulic welding table that can adjusted the height so that can fix the bad postural while operator welding the objects. 


\section{Acknowledgements}

Thanks to all those who have supproted this research, both in the manufacturing industry of this research and all the operators of the company's production floor. Laboratory of Work System and Ergonomics Major Industrial Engineering UNTIRTA.

\section{References}

1. Suma'mur. Company Hygiene and Occupational Health. Jakarta : PT Toko Gunung Agung. (1989)

2. Manuaba, A. Implementation of ergonomics to improve the quality of human resources and productivity; The material of the K3 Seminar with the theme Through culture of K3 improve the quality of human resources. Ergonomics potpourri. Vol 1. Denpasar. Departemen of Physiology. Udayana University. (1992)

3. Grandjean, E. Fitiing The Task to The Man. $4^{\text {th }}$ edition. London : Taylor \& Francis. (1993)

4. Susihono, Wahyu. Automatic Semi Cutting Machine with Attention of Ergonomic Working Aspects. Proceeding National Seminar K3 and Ergonomic Program Application at Work. University of USU. Medan. (2009)

5. Manuaba, A. The effort of ergonomic culture in PTP XXI-XXXII; Seminar Material Cultivate Ergonomics at Sugar Factory PTP XXI-XXII Surabaya. Ergonomics potpourri. Vol 1. Denpasar. Departemen of Physiology. Udayana University. (1992)

6. Susihono, W. Ergonomics in Metal Casting Industry. Serang : Untirta Press. (2016)

7. Susihono, W. Ergonomics Approach Analysis As Basis for Identification and Repairing Working Conditions in Industrial Metal Kitchen Casting System Induction; Case Study at PT. X Ceper Klaten. (2014)

8. Mulyadi. Batan, I Made Londen. Testing Prototype e Multipurpose Wheelchair. Department of Mechanical Engineering University of Muhammadiyah Sidoarjo and ITS Surabaya. East Java. (2016)

9. Proceeding National Seminar of Industrial Engineering Conference. Department of Industrial Engineering, University of Sebelas Maret. Surakarta. Hal 284-290. ISBN 978-602-70259-2-9.

10. Anizar, Suriadi Joko. Posture Analysis of Operators in Boiler Section with Ovako Working Posture Analysis System Method at PTPN V SEI Rokan Riau. Paper in the National Seminar on Industrial Engineering and Congress BKSTI V. Makassar, 16-17 Juli (2008)

11. Chapanis, A. National and Cultural Variables in Ergonomics. Ergonomics-17. P 153175. (1974) 